Repai $r$ of segment al bone defects i $n r$ abbi $t$ t i bi a promot ed by a compl ex of $\beta$ - tri cal ci um phosphate and hepat ocyte grouth fact or

\begin{tabular}{|l|l|}
\hline 著者 & $\begin{array}{l}\text { Goshi ma Keni chi, Nakase Junsuke, Xu Q ng, } \\
\text { Nat sunnt o Kuni o, Tsuchi ya H r oyuki }\end{array}$ \\
\hline 著者別表示 & 五嶋 謙一, 中瀬 順介, 松本 邦夫, 土屋 弘行 \\
\hline $\begin{array}{l}\text { j our nal or } \\
\text { publ i cat i on titl e }\end{array}$ & Journal of Orthopaedi c Sci ence \\
\hline vol une & 17 \\
\hline nunber & 5 \\
\hline page r ange & $639-648$ \\
\hline year & 2012-09-01 \\
\hline URL & ht t p: //doi . or g/10. 24517/00013760 \\
\hline
\end{tabular}




\title{
Repair of segmental bone defects in rabbit tibia promoted by a complex of $\beta$-tricalcium phosphate and hepatocyte growth factor
}

\author{
Kenichi Goshima $\cdot$ Junsuke Nakase $\cdot$ \\ Qing Xu · Kunio Matsumoto $\cdot$ Hiroyuki Tsuchiya
}

Received: 23 July 2011 / Accepted: 1 June 2012

(C) The Japanese Orthopaedic Association 2012

\begin{abstract}
Background Segmental bone defect repair remains a clinical and scientific challenge with increasing interest focused on bone tissue engineering. Clinical studies are ongoing to address application of hepatocyte growth factor (HGF) for treatment of some diseases; however, the use of HGF in bone tissue engineering has not been addressed. This study was performed to evaluate the effect of HGF in a complex of $\beta$-tricalcium phosphate ( $\beta$-TCP) and collagen in repairing segmental bone defects.

Methods Segmental bone defects $5 \mathrm{~mm}$ long were created in the middle of the tibial shafts of rabbits. The defect was stabilized with external fixators and implanted with a complex of $\beta$-TCP granules and collagen, with or without $100 \mu \mathrm{g}$ recombinant human HGF. Biweekly, bone regeneration and $\beta$-TCP resorption were assessed radiographically and histologically. At 4 and 8 weeks, bone regeneration was evaluated by use of micro-computed tomography and mechanical tests.

Results Compared with the bone tissue treated with $\beta$-TCP and collagen, mineralization, angiogenesis, new bone formation, and absorption of $\beta$-TCP were promoted 4 weeks postoperatively by treatment with HGF in the $\beta$-TCP and collagen group. These changes were associated with promoting biomechanical regeneration. By 8 weeks,
\end{abstract}

K. Goshima $(\bowtie) \cdot$ J. Nakase $\cdot$ H. Tsuchiya

Department of Orthopedic Surgery,

Graduate School of Medical Science,

Kanazawa University, 13-1 Takara-machi,

Kanazawa 920-8641, Japan

e-mail: syunsuke5552000jp@yahoo.co.jp

Q. Xu $\cdot$ K. Matsumoto

Cancer Research Institute, Kanazawa University,

Kakuma-machi, Kanazawa 920-1192, Japan the formation of bone marrow in newly generated bone and absorption of the $\beta$-TCP granules were completed in a shorter period by combining HGF with $\beta$-TCP and collagen, compared with tissues without HGF.

Conclusions The combined application of HGF in a $\beta$-TCP and collagen matrix promoted histological bone healing and augmented mechanical strength of the healing bone, particularly in the early stages. The combined use of HGF and $\beta$-TCP for treatment of bone defects made a substantial difference.

\section{Introduction}

Segmental bone defects can result from high-energy trauma, resection of bone tumors, or treatment of nonunion. Such bone defects have been clinically treated by implantation of bioceramics or autogenous and allogenous bone grafts. Although autografting is a popular procedure for reconstructive surgery, the limited sources of bone grafts, persistent pain, nerve damage, and cosmetic characteristics at the donor site are disadvantages [1]. In contrast, there are no donor site problems for allografting, but allografting has some clinical risks, for example disease transmission and immunological reactions [2].

Bone tissue engineering has attracted much attention as an approach to improve these problems. This new therapeutic technology induces bone regeneration by use of a variety of growth factors, osteogenic cells, biocompatible scaffolds, or a combination of these approaches [2, 3]. It is highly likely that a combination of osteoinductive growth factors and scaffolds provides an appropriate osteoinductive environment for osteogenic cells. Promoting bone formation by applying polypeptide growth factors has been reported, including bone morphogenetic protein (BMP) [4], 
fibroblast growth factor-2 (FGF-2) [5], platelet-derived growth factor (PDGF) [6], and vascular endothelial cell growth factor (VEGF) [7].

Among growth factors, hepatocyte growth factor (HGF), originally identified and cloned as a growth-promoting factor of mature hepatocytes [8], has biological activity including promotion of cell proliferation and migration, prevention of cell death, and induction of morphogenesis. On the basis of this biological activity, HGF and its receptor Met are involved in the development, repair, and protection of tissues [9]. Administration of HGF or expression of the HGF gene promotes tissue regeneration and improves the pathology of a variety of disease models of different tissues [9, 10]. A recent study demonstrated that administration of recombinant HGF enhances tendon healing in the bone tunnel and leads to improved biomechanical fixation within a shorter period postoperatively [11]. In a bone fracture model, local expression of the HGF gene promotes healing of bone fractures [12], and administration of HGF induces expression of the BMP receptor [13]. These results suggest a potential application of HGF as material-based regenerative medicine to facilitate repair of bones and ligaments in combination with orthopedic surgery.

Because scaffolding materials basically function as a substrate for attachment and proliferation of osteogenic cells during bone regeneration, three-dimensional biodegradable materials with a porous structure, for example glycolide-lactide copolymer nonwoven fabrics, collagen sponges, and calcium phosphate ceramics, have been used [14]. $\beta$-Tricalcium phosphate ( $\beta$-TCP) is a calcium phosphate ceramic used as an alternative bone substitute for autografts during bone grafting. It has excellent osteoconduction and resorbability when filling bone defects [15]. $\beta$-TCP is also a potential carrier for local delivery of polypeptide growth factors, including BMP-2 [16], FGF-2 [17], and PDGF [6], to promote bone healing. However, the effects of a complex of $\beta$-TCP and HGF on bone healing have yet to be addressed.

The objective of this study was to evaluate the effect of a complex of $\beta$-TCP and collagen combined with HGF on bone regeneration for segmental bone defects in a rabbit model. This is the first study to address the therapeutic effects of the $\beta$-TCP and HGF complex on bone healing.

\section{Materials and methods}

Materials

$\beta$-TCP was provided by Olympus Biomaterials (Tokyo, Japan). Fine $\beta$-TCP powder was synthesized by wet milling (a mechanochemical method). $\mathrm{CaHPO}_{4} \cdot \mathrm{H}_{2} \mathrm{O}$ and $\mathrm{CaCO}_{3}$ at a molar ratio of 2:1 were mixed into a slurry with pure water and particles of zirconium in a pot mill for $24 \mathrm{~h}$, dried at $80^{\circ} \mathrm{C}$, and calcium-deficient hydroxyapatite was obtained. This crystal was converted to $\beta$-TCP by calcination at $750{ }^{\circ} \mathrm{C}$ for $1 \mathrm{~h}$. A porous $\beta$-TCP block was obtained after sintering the $\beta$-TCP powder at $1050{ }^{\circ} \mathrm{C}$ for $1 \mathrm{~h}$. The porosity of the block was $75 \%$ with a mean macropore size of $200 \mu \mathrm{m}$. In this study, the $\beta$-TCP block was crushed in a ceramic bowl into granules ranging in diameter from 300 to $500 \mu \mathrm{m}$.

Atelocollagen, a highly purified type 1 collagen derived from calf dermis, was obtained from Koken (Tokyo, Japan). This telopeptide was digested with pepsin to reduce antigenicity. Recombinant human HGF (the five amino acid-deleted type) was obtained from Kringle Pharma (Osaka, Japan). To prepare implants, $0.2 \mathrm{~g}$-TCP granules, $0.25 \mathrm{ml} 1 \%$ collagen solution, and $100 \mu \mathrm{g}$ HGF were aseptically mixed in a dish immediately before surgery.

Animals and surgical procedure

Sixty female Japanese white rabbits, weighing between 2.5 and $3.0 \mathrm{~kg}$, were used for this experiment. The animal experiments were conducted in strict accordance with the regulations of the Institutional Animal Care and Use Committee of Kanazawa University, Japan. The rabbits were anesthetized by subcutaneous injection of ketamine hydrochloride $(35 \mathrm{mg} / \mathrm{kg}$ body weight; Sankyo Pharmaceutical, Tokyo, Japan) and xylazine $(5 \mathrm{mg} / \mathrm{kg}$ body weight; Bayer, Tokyo, Japan), and intravenous injection of pentobarbital sodium $(40-50 \mathrm{mg} / \mathrm{kg}$ body weight; Abbott Laboratories, Chicago, IL, USA). A longitudinal skin incision was made on the anteromedial aspect of the right tibia, and the periosteum was carefully stripped of surrounding soft tissue and fascia. Four half pins $2 \mathrm{~mm}$ in diameter (Stryker, Geneva, Switzerland) were inserted into the medial aspect of the tibia, perpendicular to its axis, and a unilateral external fixator of our design was applied. A segmental bone defect $5 \mathrm{~mm}$ long was created in the middle of the tibial shaft, by use of a bone saw. Muscle attachment was repaired and the skin was closed in layers.

The animals were divided into three experimental groups as follows. In group A $(n=20)$, the bone defect was filled with a complex of $\beta$-TCP granules, collagen, and HGF $(100 \mu \mathrm{g})$. The volume of the complex was slightly larger than that of the original bone defect. In group B $(n=20)$, the defect was filled with a mixture of $\beta$-TCP and collagen. In group $\mathrm{C}(n=20)$, the bone defect was left empty (Fig. 1). Six animals from each group were euthanized with an intravenous dose of sodium pentobarbital 4 and 8 weeks after surgery. After removing the external fixator and soft tissue surrounding the tibia, micro-computed tomography (CT) and biomechanical analysis was performed. 
Radiological and micro-CT analysis

After 2, 4, 6, and 8 weeks each rabbit was examined by $\mathrm{X}$-ray, under anesthesia, to evaluate new bone formation and resorption of the $\beta$-TCP granules in the bone defect. All animals had an anteroposterior and a mediolateral $\mathrm{X}$-ray of their operated tibia.

For detailed evaluation of the healing process, six rabbits in each group were examined by use of micro-CT (SMX-100CT-SV; Shimadzu, Kyoto, Japan).

Biomechanical analysis

To evaluate 4 and 8 -week bone regeneration, biomechanical testing of six tibias in both groups $\mathrm{A}$ and $\mathrm{B}$ was
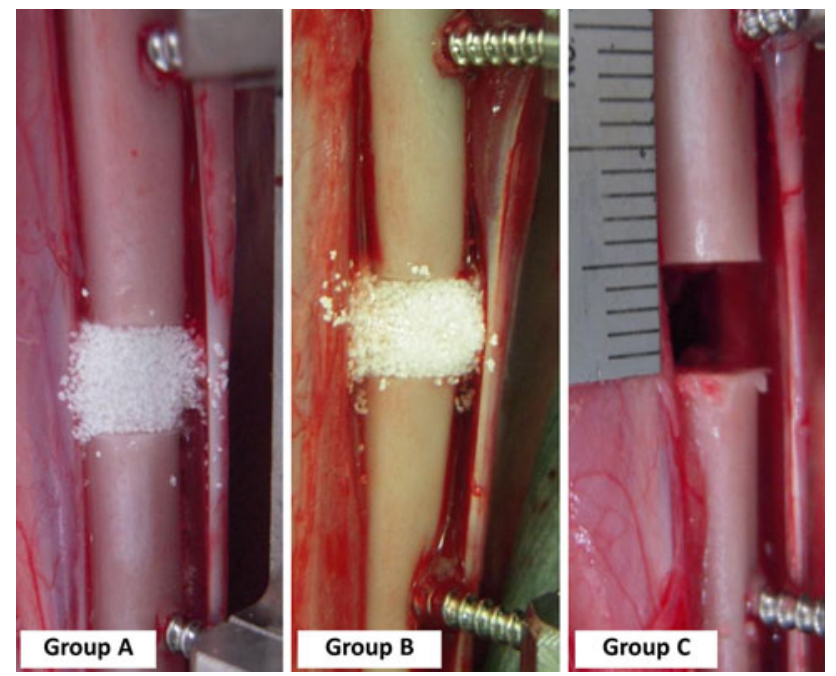

Fig. 1 Macroscopic appearance of segmental defects. A 5-mm bone defect was created in the middle of the tibia and stabilized with a unilateral external fixator. The defect was implanted with a complex of $\beta$-tricalcium phosphate ( $\beta$-TCP) granules and collagen, either with $100 \mu \mathrm{g}$ hepatocyte growth factor (HGF) (group A) or without HGF (group $B$ ). The bone defect was left empty in the control (group $C$ ) conducted. For this analysis, a three-point bending test was performed using a universal testing machine (Model 4482; Instron, Norwood, MA, USA). The maximum load (N) was interpreted and calculated from the load-deflection curve, which was displayed continuously on the computerized monitor. Mechanical data ratios of the mechanical data from the segmental bone defect tibia to the intact tibia (percentage failure load) were calculated.

\section{Histological analysis}

Two rabbits in each group were euthanized 2, 4, 6, and 8 weeks after surgery, and the histology of the osteotomy gap was studied. The specimens were rapidly fixed in $10 \%$ formalin, and conventional paraffin-embedded sections were prepared. The specimens were sectioned at $5-\mu \mathrm{m}$ thickness parallel to the bone axis and stained with hematoxylin and eosin.

Semi-quantitative evaluation of -TCP area and new bone formation

New bone formation and the $\beta$-TCP remaining in the defect were quantified by color-based thresholding and use of image-analysis software (WinRoof; Mitani, Tokyo, Japan). We colored the areas of new bone formation in pink and those with $\beta$-TCP in blue. A square of side $2 \mathrm{~mm}$ in the bone defect was defined as the total area, and area rates of new bone formation and $\beta$-TCP were calculated relative to this total area (Fig. 2).

\section{Statistical analysis}

All data are expressed as the mean \pm SD. Statistical analysis was performed by use of Student's $t$ test. Values of $p<0.05$ were considered statistically significant.
Fig. 2 Representative image for semi-quantitative evaluation of the $\beta$-tricalcium phosphate $(\beta-T C P)$ and new bone formation areas. In tissue sections subjected to $\mathrm{H} \& \mathrm{E}$ staining, a square of side of $2 \mathrm{~mm}$ was defined as the total area, and the areas of new bone formation and of $\beta$-TCP were calculated and expressed as a percentage of this total area

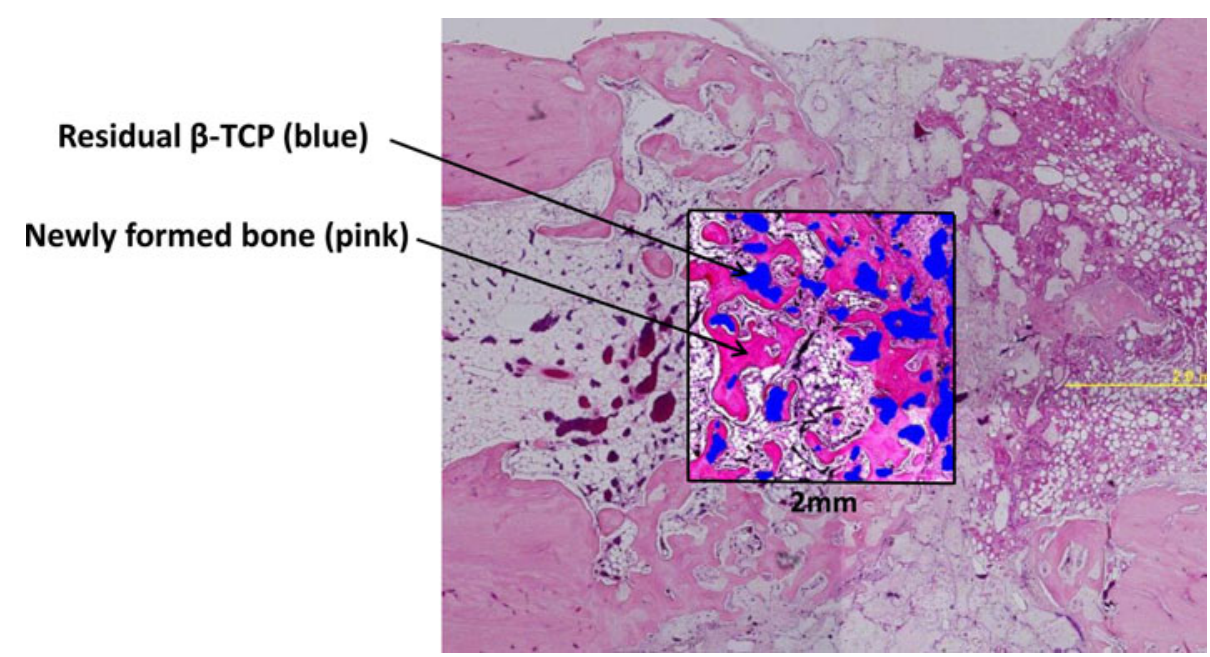




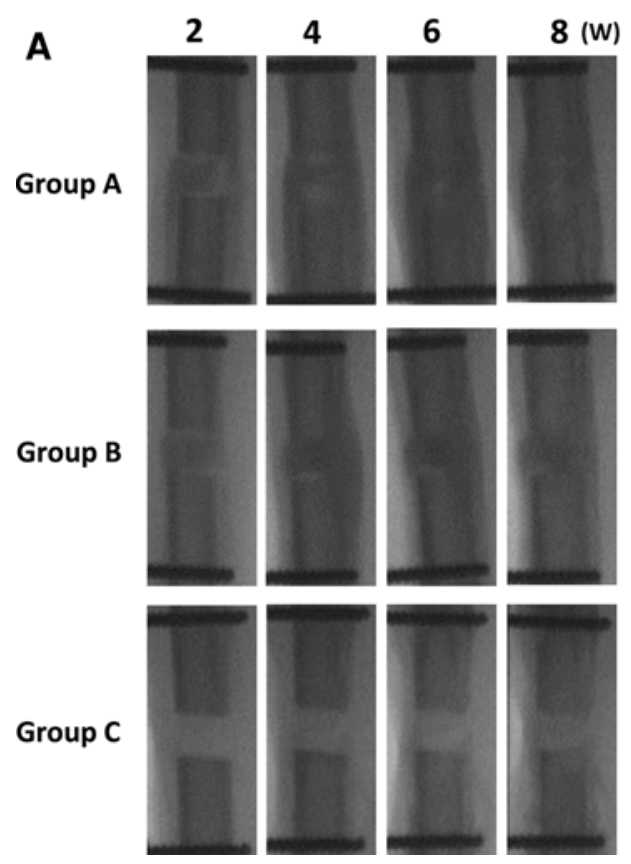

Fig. 3 Change in the healing processes of segmental bone defects as analyzed by anteroposterior (a) and mediolateral (b) radiographic images. Group A were treated with a mixture of $\beta$-tricalcium phosphate $(\beta$-TCP), collagen, and hepatocyte growth factor (HGF)

\section{Results}

Changes in radiological, macroscopic, and micro-CT findings

First, the defect regions were analyzed by radiography to evaluate healing (Fig. 3a, b). Twenty rabbits were used in each experimental group; eight rabbits in group $\mathrm{C}$ were excluded from subsequent experiments because of breakage at the defect site owing to delayed union. The other 12 rabbits in group $\mathrm{C}$ were used for radiological examination only.

In the untreated control (group C), substantial detectable changes were not seen within 4 weeks on both the anteroposterior (Fig. 3a) and mediolateral (Fig. 3b) radiographic images. Marginal changes were observed at 6 weeks on the mediolateral image and only a small amount of bone formation was observed at the two ends at 8 weeks. Clearly, no solid union was achieved in group $\mathrm{C}$ by 8 weeks postoperatively. In contrast, a clear increase in callus density and volume was observed in group A ( $\beta$-TCP, collagen, and HGF) compared with group B ( $\beta$-TCP and collagen) at 2 and 4 weeks. Anteroposterior and mediolateral radiographic images indicated that synostosis was almost complete 8 postoperatively in groups A and $\mathrm{B}$. $\beta$-TCP granules were clearly visible with most remaining within the defect in groups A and B at 2 weeks. In group A, the margins of

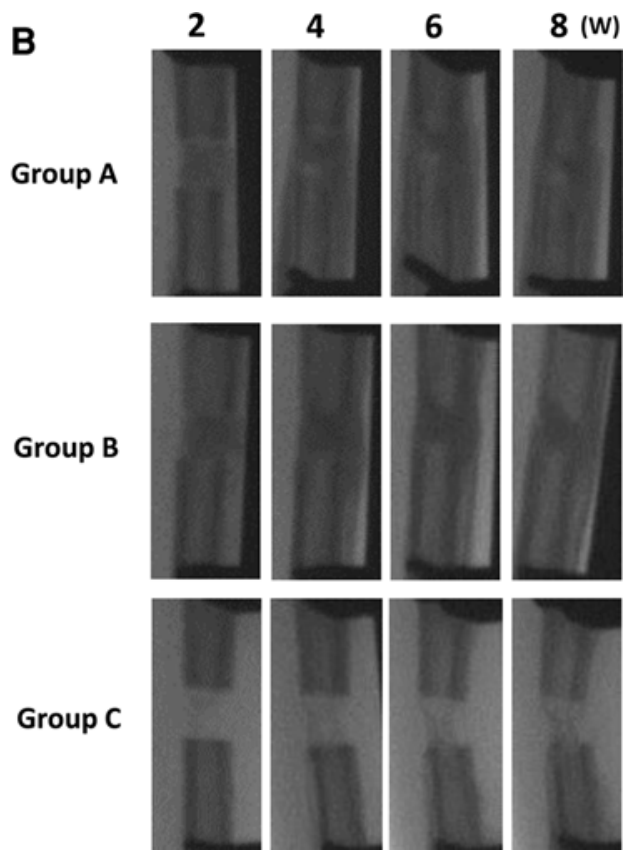

$(n=20)$; group B were treated with $\beta$-TCP and collagen $(n=20)$; group $\mathrm{C}$ were untreated $(n=12)$. Each image is representative of each group. Eight rabbits in group $\mathrm{C}$ were excluded from the analysis because of impaired union and breakage at the defect site

the $\beta$-TCP granules became unclear 4 weeks postoperatively, indicating their absorption. Thereafter, the $\beta$-TCP granules became time-dependently coarser, and most of the $\beta$-TCP granules disappeared and were replaced by bone at 8 weeks. However, in group $\mathrm{B}$, absorption of $\beta$-TCP granules was delayed compared to that in group A with most of the $\beta$-TCP granules remaining at the end of 8 weeks in group B.

The macroscopic appearance of the segmental bone defect lesions indicated that healing of the segmental defect was almost complete in group $\mathrm{A}$, whereas bone healing seemed to be achieved but residual $\beta$-TCP granules remained in group B (Fig. 4).

Consistent with the changes detected by radiographic and macroscopic observation, the micro-CT analysis indicated that a solid union had formed in groups $\mathrm{A}$ and $\mathrm{B}$ at 8 weeks. At 4 weeks, $\beta$-TCP granules in group A were coarser than those of group B. At 8 weeks, most of the $\beta$-TCP granules had disappeared and were replaced by bone in group $\mathrm{A}$, whereas most of the $\beta$-TCP granules remained in group B (Fig. 5). Taken together, these results indicate that regeneration of segmental bone defect is promoted by combinations of $\beta$-TCP and collagen (group B) and $\beta$-TCP granules, collagen, and HGF (group A), whereas overall regeneration, including absorption of $\beta$-TCP granules, seemed to be best promoted by the $\beta$-TCP, collagen, and HGF matrix. 


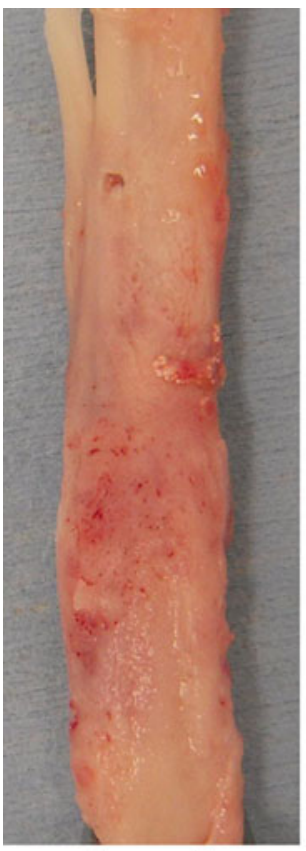

Group A

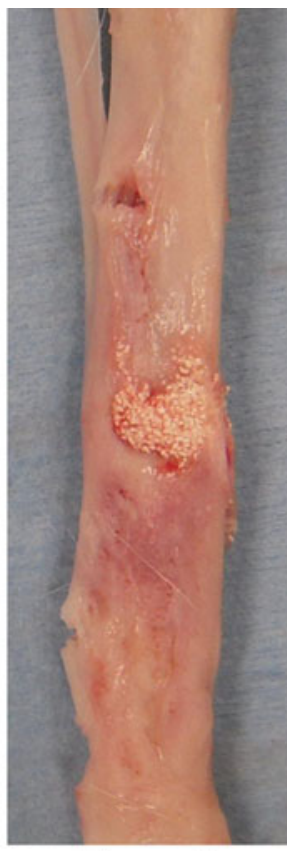

Group B
Fig. 4 Macroscopic appearance of the defects 8 weeks postoperatively. Group A were treated with a mixture of $\beta$-tricalcium phosphate ( $\beta$-TCP), collagen, and hepatocyte growth factor (HGF); group B were treated with $\beta$-TCP and collagen. These photographs are representative of each experimental group
4 weeks
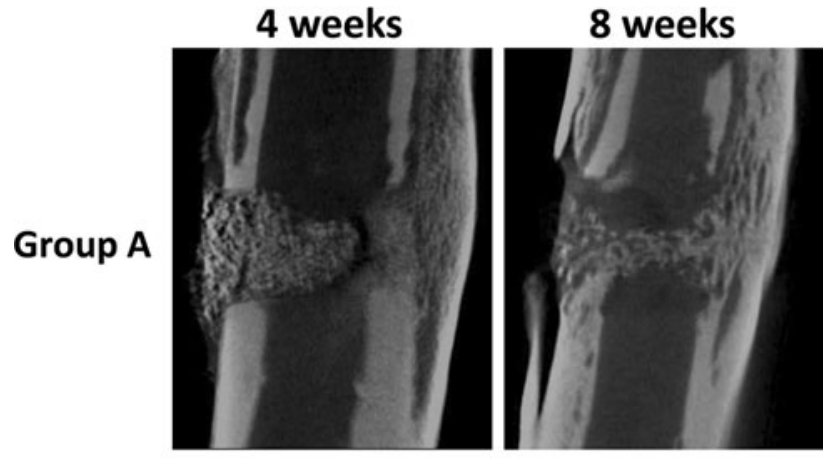

Group B
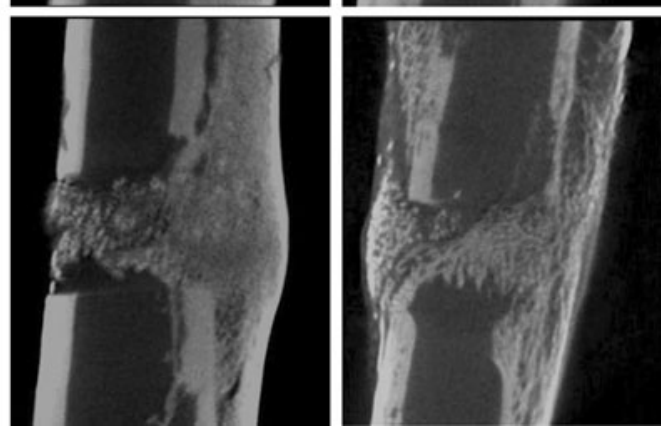

Fig. 5 Coronal view of the central defects by micro-computed tomography (CT) at 4 and 8 weeks postoperatively. Group A were treated with a mixture of $\beta$-tricalcium phosphate $(\beta$-TCP), collagen, and hepatocyte growth factor (HGF); group B were treated with $\beta$-TCP and collagen. Six rabbits in each group were examined by use of micro-CT and representative photographs in each group are shown
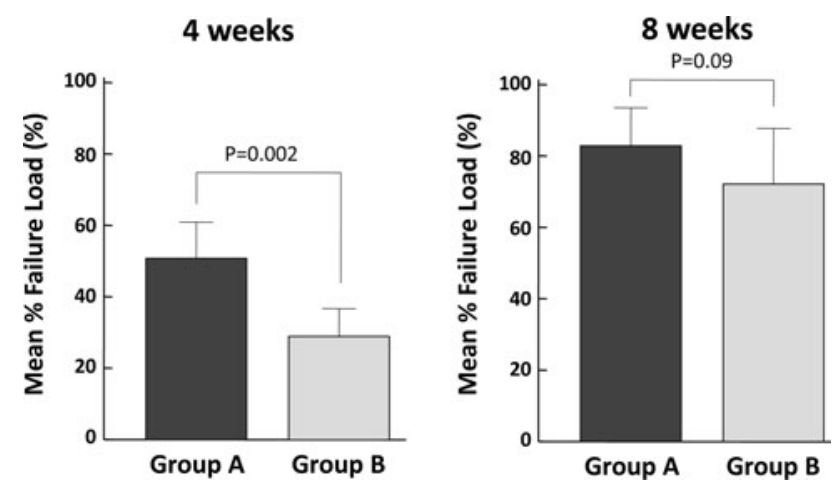

Fig. 6 Change in biomechanical strength of segmental bone defect sites at 4 and 8 weeks postoperatively. Group A were treated with a mixture of $\beta$-tricalcium phosphate ( $\beta$-TCP), collagen, and hepatocyte growth factor (HGF) $(n=20)$; group B were treated with $\beta$-TCP and collagen $(n=20)$. Because union of the bone defect was impaired at 8 weeks in the untreated control group, they were excluded from this analysis

\section{Biomechanical analysis}

The legs were subjected to biomechanical examination to determine whether facilitated regeneration of the segmental bone defects by application of additional HGF was associated with functional regeneration (Fig. 6). Four weeks postoperatively, the mean ratio of failure loads in group A was significantly greater $(p=0.002)$ than that of group B (50.9 \pm 12.5 vs. $29.1 \pm 8.0 \%$, respectively). Although no significant differences were observed, the mean ratio of failure loads in group A tended to be slightly greater than that in group B at 8 weeks $(83.3 \pm 10.4 \%$ in group A vs. $72.7 \pm 15.2 \%$ in group $\mathrm{B} ; p=0.09$ ).

\section{Histological changes}

Two weeks postoperatively, fibroblast-like cells characteristic of granulation tissue, newly formed blood vessels, and monocytes and/or inflammatory cells infiltrated into the $\beta$-TCP micropores, and a small amount of immature bone formation was observed around the $\beta$-TCP in group A. In group B, fibroblast-like cells appeared around the $\beta$-TCP, but angiogenesis was less than that in group A, and immature bone formation was not seen (Fig. 7a). Four weeks postoperatively, granulation tissue in the $\beta$-TCP micropores underwent mineralization, and the appearance of multinucleated giant cells characteristic of osteoclasts seemed to be associated with absorption of $\beta$-TCP, and new bone formation was associated with angiogenesis in group A. In group B, fibroblast-like cells expanded, and angiogenesis and immature bone formation was seen to a lesser extent than in group A. Absorption of $\beta$-TCP was unclear. Thus, histological changes toward healing of the bone defect in group A 
Fig. 7 Histological changes during healing of the segmental bone defects. Tissue sections were stained with H\&E. Group A were treated with a mixture of $\beta$-tricalcium phosphate $(\beta$-TCP), collagen, and hepatocyte growth factor (HGF) $(n=20)$; group B were treated with $\beta$-TCP and collagen $(n=20)$. Representative longitudinal histological sections of both groups $2,4,6$, and 8 weeks after surgery are shown
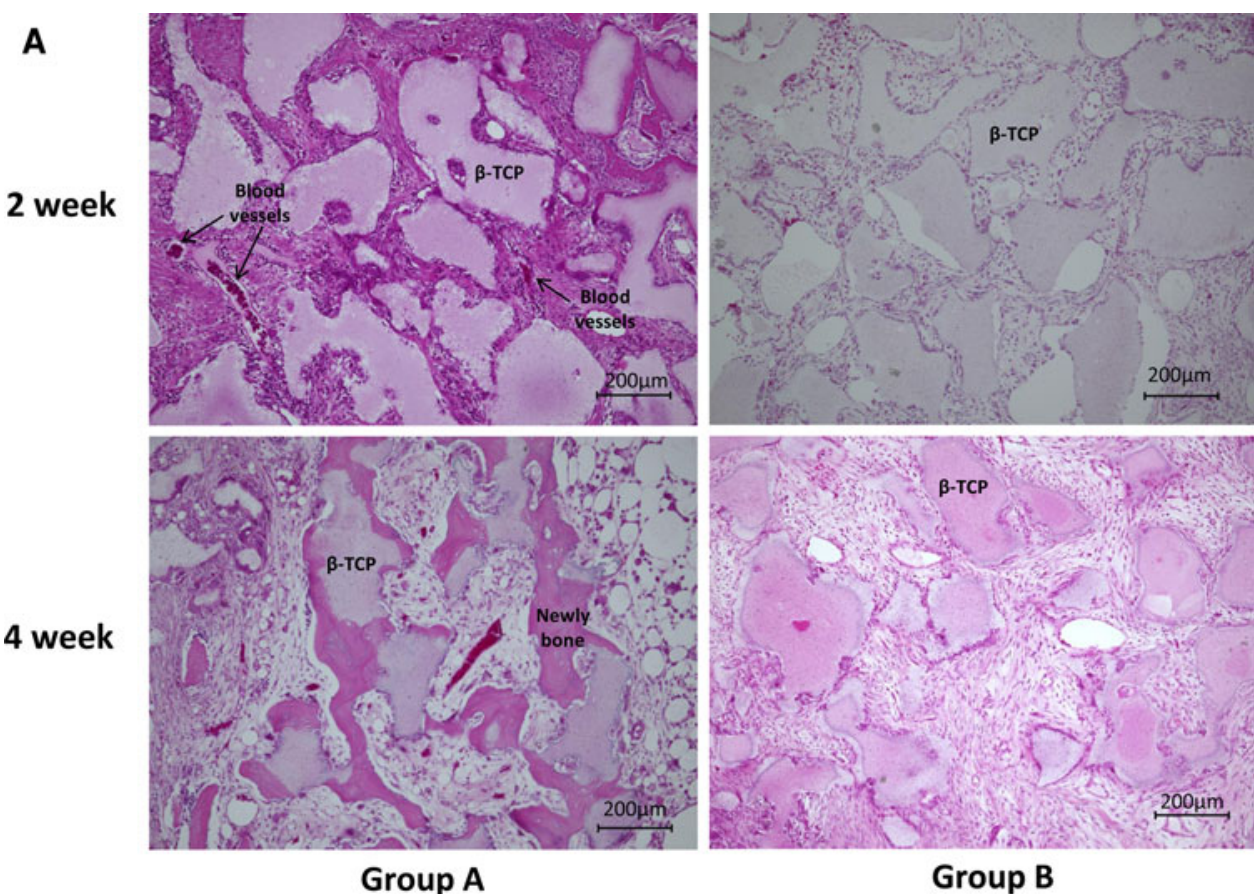

\section{Group B}

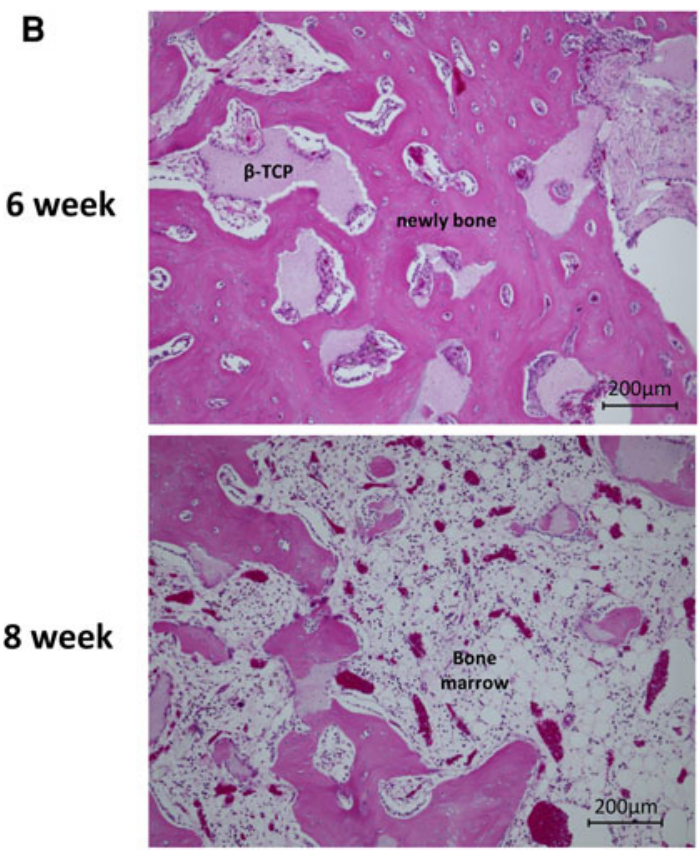

Group A

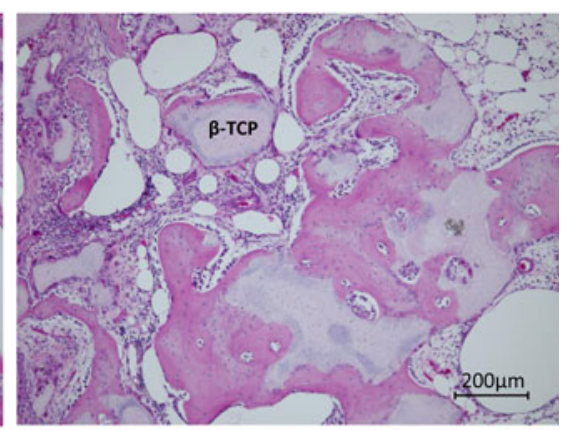

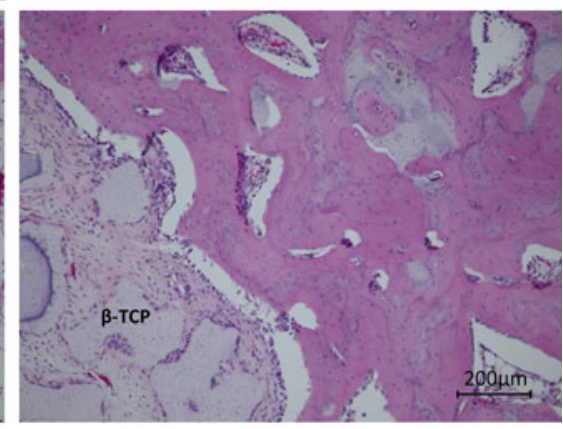

Group B took less time than in group B (Fig. 7a). Six weeks postoperatively, multiple islands of newly formed bone were integrated, and woven bone structures clearly appeared in group A. Absorption of $\beta$-TCP had progressed further than at 4 weeks. In group B, new bone formation appeared, and this was associated with angiogenesis and absorption of $\beta$-TCP (Fig. 7b).

After 8 weeks, newly generated bone was replaced by bone marrow tissue, and the $\beta$-TCP granules were mostly absorbed and had disappeared in group A. In group B, new bone formation had progressed further after 6 weeks, and woven bone structures had appeared. Thus, although histological changes similar to those in group A occurred in group B, the amount and rate of new bone formation was less and slower than that of group A. Absorption of $\beta$-TCP granules occurred but some granules still remained at the end of 8 weeks in group B (Fig. 7b). 
Measurement of newly formed bone and residual $\beta$-TCP area

The area rates of new bone formation in groups $\mathrm{A}$ and $\mathrm{B}$ were, respectively, 2.5 and $0.4 \%$ at 2 weeks, 19.2 and $10.4 \%$ at 4 weeks, and 28.8 and $25.9 \%$ at 6 weeks. The area rate of new bone formation in group A was larger than that of group B at 2, 4, and 6 weeks. However, at 8 weeks, the area rate of new bone formation in group A was smaller than that of group B (36.0 vs. $42.3 \%)$. No statistical difference was observed between the groups at any time (Fig. 8a). The area rates of residual $\beta$-TCP in groups A and B were, respectively, 41.3 and $51.1 \%$ at 2 weeks, 18.1 and $31.6 \%$ at 4 weeks, 14.1 and $19.6 \%$ at 6 weeks, and 6.1 and $11.4 \%$ at 8 weeks. The area rate of residual $\beta$-TCP in group A was smaller than that of group B at any time period. Although the area rate of residual $\beta$-TCP in group A was significantly smaller than that of group $B$ at
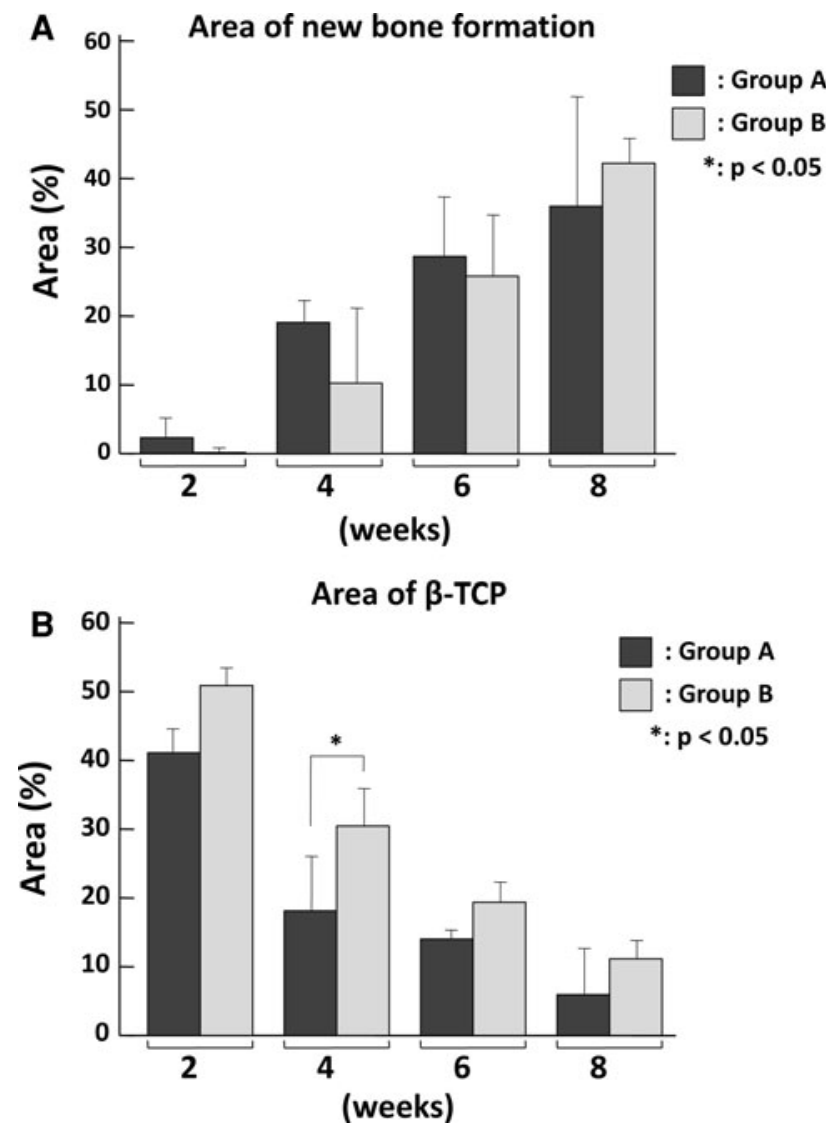

Fig. 8 Changes in areas of new bone formation (a) and the remaining $\beta$-tricalcium phosphate $(\beta$-TCP) (b). Group A were treated with a mixture of $\beta$-TCP, collagen, and hepatocyte growth factor (HGF) $(n=20)$; group B were treated with $\beta$-TCP and collagen $(n=20)$. The areas of new bone formation and $\beta$-TCP were quantified using color-based thresholding and image-analysis software (WinRoof) and expressed as a percentage of the area analyzed. A representative photograph for determination of these areas is shown in Fig. 2
4 weeks, no statistical difference was observed between the groups at other time periods (Fig. 8b).

\section{Discussion}

Extracellular scaffold proteins, bioactive substances, and osteogenic progenitor cells induce bone formation or promote osteogenesis by enhancing osteogenic cell migration, proliferation, and differentiation [3]. In tissue engineering, incorporation of one or more of the fundamental elements needed to regenerate bone tissue, for example scaffold proteins, bioactive substances, or osteogenic progenitor cells into the target site is the basic strategy used to promote bone regeneration in situ. In this study, we demonstrated that the complex of human recombinant HGF, $\beta$-TCP, and collagen promoted bone healing in a rabbit model of segmental bone defects and increased the mechanical strength of the bone defect in rabbit, particularly at an early stage.

Previous studies have demonstrated therapeutic actions of HGF in different disease models in different tissues, including the liver, kidney, lung, and nervous tissue [9, 18]. Consistently, tissue-selective disruption of the Met gene in the liver, kidney, or epidermis indicated impairment in regeneration and healing and/or an increase in epithelial apoptosis [26, 27]. Compared with understanding of the physiological functions of HGF in tissues originating from endodermal and epithelial cells, for example the liver and nervous tissue, the physiological function and biological activity of HGF during bone metabolism and regeneration have yet to be addressed, perhaps because early studies indicated that the Met receptor is expressed in a variety of epithelial cells. However, studies conducted in the past several years have revealed biological activity of the HGFMet pathway during bone metabolism and fracture healing. HGF and its receptor, c-Met, are expressed in both osteoblasts and osteoclasts [19]. HGF, in combination with vitamin D3, promotes growth and differentiation of human mesenchymal cells into osteogenic cells [20]. HGF enhances differentiation of osteoblasts [21] and induces the expression of BMP receptors during the early phase of fracture repair [13].

Biocompatibility, osteoconductivity, an interconnected porous structure enabling neovascularization, mechanical strength, and biodegradability are appropriate characteristics of scaffold materials for bone regeneration [22]. $\beta$-TCP and hydroxyapatite are osteoconductive and biocompatible with bone tissue, whereas $\beta$-TCP, but not hydroxyapatite, is biodegradable. A paste-like composite of $\beta$-TCP granules and collagen, which we used in this study, is applicable to defects of any shape and is easy to handle. In geriatric osteoporotic rats, PDGF-BB in a mixture of 
$\beta$-TCP and collagen promotes healing of bone fractures after a tibial osteotomy, compared with the material without PDGF-BB [6]. In rabbits, FGF-2 in a mixture of $\beta$-TCP and collagen facilitates healing of segmental bone defects [17]. BMP-2 in gelatin-based sponges containing $\beta$-TCP has higher osteoinductive activity at bone-defect sites than sponges that do not contain BMP-2 [16]. The results presented in this paper indicate that HGF in a complex of $\beta$-TCP and collagen added to tibial osteotomy gaps effectively promoted bone repair and $\beta$-TCP absorption. Taken together, these polypeptide growth factors potentiate the effectiveness of $\beta$-TCP for healing bone defects in less time and with better biomechanical quality than $\beta$-TCP alone, whereas $\beta$-TCP provides a delivery system for polypeptide growth factors, thereby promoting the osteoinductive environment.

HGF has strong angiogenic properties [10, 23], and neovascularization is fundamental for bone development and regeneration, supplying blood cells, oxygen, minerals, ions, glucose, hormones, and growth factors [25]. For osteoblasts and osteoclasts, HGF stimulates proliferation of osteoblasts and chemotactic migration and replication of osteoclasts [24]. In this study, we showed that along with the activation of new bone formation, more extensive blood vessels were formed in bone defects to which HGF was applied than those without HGF. $\beta$-TCP absorption was much accelerated in the defective regions in the HGFtreated group compared to the group without HGF. Eight weeks after surgery, most of the $\beta$-TCP was absorbed and replaced by newly formed bone in the HGF-treated group, whereas $\beta$-TCP granules remained in defected regions without HGF. Previous studies indicated that osteoclasts are of major importance in bioresorption of $\beta$-TCP [28]. Inhibition of osteoblast formation resulted in reduced $\beta$-TCP resorption and bone formation, suggesting a coupling between osteoclast-mediated $\beta$-TCP resorption and bone formation [29]. Taken together, we assume that HGFinduced angiogenesis, mesenchymal cell proliferation, and osteoclasts' migration and proliferation were responsible for promoting bone formation, the appearance of osteoclasts around the $\beta$-TCP, and facilitated $\beta$-TCP resorption.

The histological analysis revealed that HGF promoted new bone formation in defective regions and this was associated with recovery of biomechanical strength during the early stages of bone regeneration within 4 weeks. No significant differences in biomechanical recovery were observed between the groups with or without HGF 8 weeks postoperatively. The biological activity of HGF such as angiogenesis and promotion of growth of mesenchymal progenitor cells and their differentiation into osteoblasts is more important in the early stages of bone regeneration than in the late stages. HGF contributes to fracture repair by inducing BMP receptor expression during the early stage of the repair [13]. Although significant reduction in biological activity of HGF in solution at $37{ }^{\circ} \mathrm{C}$ is not seen at least for 1 week (unpublished data), at the injury site HGF might be potentially inactivated because of several proteases, for example elastase, which is secreted by inflammatory cells. Thus, HGF has biological activity that is important in the early stage of bone healing, rather than the late stages, thereby leading to rapid histological and biomechanical healing within 4 weeks. Additionally, it seemed likely that release and delivery of HGF from the complex of $\beta$-TCP and collagen may occur earlier than the biological degradation of $\beta$-TCP, thereby enabling HGF to exert its biological actions during the early stages of bone regeneration.

Our results suggest the potential application of HGF for treating bone defects or bone fractures; however, several issues should be addressed before clinical application. HGF has substantial affinity for different types of collagen and collagen-bound HGF is biologically active [30]. Thus, the mixture of type- 1 collagen and $\beta$-TCP was expected to provide a biodegradable scaffold for local delivery of HGF. On the other hand, previous studies using local administration of HGF in various models in different species including rabbit revealed significant therapeutic effects of $100 \mu \mathrm{g}$ HGF [11, 31-34]. Therefore, we selected a dose of $100 \mu \mathrm{g}$ HGF/injection for the HGF/ $\beta$-TCP/collagen complex because HGF was expected to exert biological effects and we could evaluate if HGF would have therapeutic benefits in this rabbit model. However, the chemical stability and pharmacological availability of HGF released from the HGF/ $\beta$-TCP/collagen complex, and the optimum dose of HGF, should be determined in subsequent preclinical studies before future clinical studies. Likewise, the potential risk of stimulation of premalignant lesions by HGF should be tested. Nevertheless, we have obtained evidence, for the first time, that recombinant human HGF combined with $\beta$-TCP and collagen promoted bone healing and increased biomechanical strength of a bone defect in rabbits, particularly at an early stage. HGF and $\beta$-TCP could shorten the period for bone healing and fusion and could be implanted during a single surgery, perhaps without preparation of bone for an autograft, thereby reducing surgical time. Overall, these changes are expected to improve the physical and mental well-being of patients.

\section{Conclusion}

In a rabbit osteotomy model, HGF combined with $\beta$-TCP and collagen in a matrix promoted bone healing and increased mechanical strength, particularly at the early stage of healing. 
Conflict of interest The authors did not receive and will not receive any benefits or funding from any commercial party related directly or indirectly to the subject of this article.

\section{References}

1. Cockin J. Autologous bone grafting; complications at the donor site. J Bone Joint Surg Br. 1971;53:153.

2. Langer R, Vacanti JP. Tissue engineering. Science. 1993;260; 920-6.

3. Petite H, Viateau V, Bensaïd W, Meunier A, de Pollak C, Bourguignon M, Oudina K, Sedel L, Guillemin G. Tissue engineered bone regeneration. Nat Biotechnol. 2000;18:959-63.

4. Einhorn TA, Majeska RJ, Mohaideen A, Kagel EM, Bouxsein ML, Turek TJ, Wozney JM. A single percutaneous injection of recombinant human bone morphogenetic protein-2 accelerates fracture repair. J Bone Joint Surg Am. 2003;85:1425-35.

5. Nakamura $Y$, Tensho $K$, Nakaya $H$, Nawata $M$, Okabe $T$, Wakitani S. Low dose fibroblast growth factor-2 (FGF-2) enhances bone morphogenetic protein-2 (BMP-2)-induced ectopic bone formation in mice. Bone. 2005;36:399-407.

6. Hollinger JO, Onikepe AO, Mackrell J, Einhorn T, Bradica G, Lynch S, Hart CE. Accelerated fracture healing in the geriatric, osteoporotic rat with recombinant human platelet-derived growth factor-BB and an injectable beta-tricalcium phosphate/collagen matrix. J Ortho Res. 2008;26:83-90.

7. Samee M, Kasugai S, Kondo H, Ohya K, Shimokawa H, Kuroda S. Bone morphogenetic protein-2 (BMP-2) and vascular endothelial growth factor (VEGF) transfection to human periosteal cells enhances osteoblast differentiation and bone formation. J Pharmacol Sci. 2008;108:18-31.

8. Nakamura T, Nishizawa T, Hagiya M, Seki T, Shimonishi M, Sugimura A, Tashiro K, Shimizu S. Molecular cloning and expression of human hepatocyte growth factor. Nature. 1989;342: $440-3$.

9. Nakamura T, Sakai K, Nakamura T, Matsumoto K. Hepatocyte growth factor twenty years on: much more than a growth factor. J Gastroenterol Hepatol. 2011;26:188-202.

10. Shigematsu H, Yasuda K, Iwai T, Sasajima T, Ishimaru S, Ohashi Y, Yamagushi T, Ogihara T, Morishita R. Randomized, doubleblind, placebo-controlled clinical trial of hepatocyte growth factor plasmid for critical limb ischemia. Gene Ther. 2010;17(9): 1152-61.

11. Nakase J, Kitaoka K, Matsumoto K, Tomita K. Facilitated tendon-bone healing by local delivery of recombinant hepatocyte growth factor in rabbits. Arthroscopy. 2010;26:84-90.

12. Matsubara H, Tsuchiya H, Watanabe K, Takeuchi A, Tomita K. Percutaneous nonviral delivery of hepatocyte growth factor in an osteotomy gap promotes bone repair in rabbits: a preliminary study. Clin Orthop Relat Res. 2008;466:2962-72.

13. Imai $Y$, Terai H, Nomura-Furuwatari C, Mizuno S, Matsumoto K, Nakamura T, Takaoka K. Hepatocyte growth factor contributes to fracture repair by upregulating the expression of BMP receptors. J Bone Miner Res. 2005;20:1723-30.

14. Bauer TW, Smith ST. Bioactive materials in orthopedic surgery: overview and regulatory considerations. Clin Orthop Relat Res. 2002;395:11-22.

15. Kondo N, Ogose A, Tokunaga K, Ito T, Arai K, Kudo N, Inoue H, Irie $\mathrm{H}$, Endo $\mathrm{N}$. Bone formation and resorption of highly purified $\beta$-tricalcium phosphate in the rat femoral condyle. Biomaterials. 2005;26:5600-8.

16. Matsumoto G, Omi Y, Kubota E, Ozono S, Tsuzuki H, Kinoshita Y, Yamamoto M, Tabata Y. Enhanced regeneration of critical bone defects using a biodegradable gelatin sponge and $\beta$-tricalcium phosphate with bone morphogenetic protein-2. J Biomater Appl. 2009;24:327-42.

17. Komaki H, Tanaka T, Chazono M, Kikuchi T. Repair of segmental bone defects in rabbit tibiae using a complex of $\beta$-tricalcium phosphate, type I collagen, and fibroblast growth factor-2. Biomaterials. 2006;27:5118-26.

18. Matsumoto K, Nakamura T. Hepatocyte growth factor: renotropic role and potential therapeutics for renal diseases. Kidney Int. 2001;59:2023-38.

19. Sato T, Hakeda Y, Yamaguchi Y, Mano H, Tezuka K, Matsumoto K, Nakamura T, Mori Y, Yoshizawa K, Sumitani K, et al. Hepatocyte growth factor is involved in formation of osteoclast-like cells mediated by clonal stromal cells (MC3T3G2/PA6). J Cell Physiol. 1995;164:197-204.

20. D'lppolito G, Schiller PC, Perez-stable C, Balkan W, Roos BA, Howard GA. Cooperative actions of hepatocyte growth factor and 1,25-dihydroxyvitamin D3 in osteoblastic differentiation of human vertebral bone marrow stromal cells. Bone. 2002;31: 269-75.

21. Hossain M, Irwin R, Baumann MJ, McCabe LR. Hepatocyte growth factor (HGF) adsorption kinetics and enhancement of osteoblast differentiation on hydroxyapatite surfaces. Biomaterials. 2005;26:2595-602.

22. Mastrogiacomo M, Muraglia A, Komlev V, Peyrin F, Rustichelli F, Crovace A, Cancedda R. Tissue engineering of bone: search for a better scaffold. Orthod Craniofac Res. 2005;8:277-84.

23. Sengupta S, Gherardi E, Sellers LA, Wood JM, Sasisekharan R, Fan TP. Hepatocyte growth factor/scatter factor can induce angiogenesis independently of vascular endothelial growth factor. Arterioscler Thromb Vasc Biol. 2003;23:69-75.

24. Grano M, Galimi F, Zambonin G, Colucci S, Cottone E, Zallone AZ, Comoglio PM. Hepatocyte growth factor is a coupling factor for osteoclasts and osteoblasts in vitro. Proc Natl Acad Sci USA. 1996;93:7644-8.

25. Kanczler JM, Oreffo RO. Osteogenesis and angiogenesis: the potential for engineering bone. Eur Cell Mater. 2008;15:100-14.

26. Huh CG, Factor VM, Sánchez A, Uchida K, Conner EA, Thorgeirsson SS. Hepatocyte growth factor/c-met signaling pathway is required for efficient liver regeneration and repair. Proc Natl Acad Sci USA. 2004;101:4477-82.

27. Dai C, Saleem MA, Holzman LB, Mathieson P, Liu Y. Hepatocyte growth factor signaling ameliorates podocyte injury and proteinuria. Kidney Int. 2010;77:962-73.

28. Chazono M, Tanaka T, Komaki H, Fujii K. Bone formation and bioresorption after implantation of injectable beta-tricalcium phosphate granules-hyaluronate complex in rabbit bone defects. J Biomed Mater Res A. 2004;70:542-9.

29. Tanaka T, Saito M, Chazono M, Kumagae Y, Kikuchi T, Kitasato $S$, Marumo K. Effects of alendronate on bone formation and osteoclastic resorption after implantation of beta-tricalcium phosphate. J Biomed Mater Res A. 2010;93:469-74.

30. Schuppan D, Schmid M, Somasundaram R, Ackermann R, Ruehl M, Nakamura T, Riecken EO. Collagens in the liver extracellular matrix bind hepatocyte growth factor. Gastroenterology. 1998; 114:139-52.

31. Okura Y, Arimoto H, Tanuma N, Matsumoto K, Nakamura T, Yamashima T, Miyazawa T, Matsumoto Y. Analysis of neurotrophic effects of hepatocyte growth factor in the adult hypoglossal nerve axotomy model. Eur J Neurosci. 1999;11:4139-44.

32. Tönges L, Ostendorf T, Lamballe F, Genestine M, Dono R, Koch JC, Bähr M, Maina F, Lingor P. Hepatocyte growth factor protects retinal ganglion cells by increasing neuronal survival and axonal regeneration in vitro and in vivo. J Neurochem. 2011; 117:892-903.

33. Ishigaki A, Aoki $M$, Nagai $M$, Warita $H$, Kato $S$, Kato $M$, Nakamura T, Funakoshi H, Itoyama Y. Intrathecal delivery of 
hepatocyte growth factor from amyotrophic lateral sclerosis onset suppresses disease progression in rat amyotrophic lateral sclerosis model. J Neuropathol Exp Neurol. 2007;66:1037-44.
34. Ueshima K, Kitaoka K, Nakase J, Xu Q, Matsumoto K, Tsuchiya H. Promotion of rabbit ligament healing by local delivery of hepatocyte growth factor. J Orthop Sci. 2011;16:451-7. 